\title{
Generalist Scholarship and Engineering Education
}

\author{
Thomas M. Adams \\ Rose-Hulman Institute of Technology \\ 5500 Wabash Ave. Terre Haute, IN, USA \\ adams1@rose-hulman.edu
}

\begin{abstract}
Generalist scholarship may be thought of as an approach to research in which simple models and methods are intentionally employed in order to ascertain trends, correlations, and high-level concepts that are difficult to discern when relying solely on more specialized methods. In the twenty-first century, however, the advent of sophisticated software and continually increasing computational power have generally led engineering - practice, research, and education alike - away from broad based analysis and towards more and more specialized techniques. And though specialized research can deliver a high degree of detail and quantitative accuracy in modeling systems and processes, physical insights and high-level connections are increasingly in peril of going overlooked. In this talk, we explore the tremendous opportunities that exist for generalist scholarship to complement specialist research with a particular emphasis on the thermalfluid sciences. Furthermore, given that the tools that characterize the methods of generalist scholarship are acquired early in a technical education, we will examine the potential to enhance engineering education through research at the generalist level, as well as to fill the pipeline of future engineering scholars.
\end{abstract}

